\title{
Electron Collision Rate in Ultrashort Laser - Metal Interaction Inferred from Reflectivity Measurements
}

\author{
T. Genieys, M. Sentis, and O. Utéza* \\ Aix-Marseille University, CNRS, LP3 UMR 7341, F-13288 Marseille, France \\ ${ }^{*}$ Corresponding author's e-mail: olivier.uteza@univ-amu.fr
}

\begin{abstract}
We study optical laser coupling of four metals (aluminum, copper, nickel and tungsten) upon single ultrashort 30-fs laser irradiation. Using dedicated experimental methodology, we carefully monitor the evolution of the material reflectivity integrated over the femtosecond pulse duration. We further apply the Drude-Lorentz formalism to infer the evolution of the effective electron collision rate $v_{\text {eff }}$ as a function of applied fluence. The knowledge of this parameter is of paramount importance in any laser - matter interaction situation because of its immediate impact on laser energy coupling in material and on its subsequent macroscopic transformation.
\end{abstract}

DOI: 10.2961/jlmn.2021.02.2010

Keywords: ultrashort laser, metals, laser-induced ablation threshold, optical laser coupling, reflectivity, effective electron collision rate

\section{Introduction}

Laser interaction in ultrashort femtosecond regime has many scientific and technical merits in fundamental research and industry [1]. This comes from the ability of femtosecond lasers to machine materials with precision and high resolution, and minimized thermal budget and collateral effects [1,2]. Nevertheless, evaluation of laser-matter interaction observables and material parameters to benchmark and guide matter transformation are still scarce in the ultrashort regime. In this context, we recently determined single-shot laser-induced ablation threshold fluence $\mathrm{F}_{\text {th }}$ for transition and post-transition metals for femtosecond pulse durations as short as 15 fs [3,4]. This brought complementary information to those already obtained at longer femtosecond pulse duration, or those often made in the multipulse regime in order to evaluate incubation effects [5-8]. All those data are in particular important in the context of micromachining of metals, encompassing surface drilling, engraving and structuring for various scientific and industrial applications $[1,9,10]$.

In this paper, we use optical reflectometry to measure the strength of laser optical coupling at the surface of different metals and Drude-Lorentz framework for theoretical description of ultrashort laser heating of metals. Our motivation is to infer the evolution of the effective electron collision rate $v_{\text {eff }}$ as a function of applied fluence. We thus provide the knowledge of an essential marker of lasermatter interaction in ultrashort regime over an extremely wide range of laser energy excitation $\left(0.1-10 \mathrm{~J} / \mathrm{cm}^{2}\right)$. Note that previous works [see for instance 11-20] mostly addressed this problematic for laser pulse duration much superior to the pulse duration (30 fs) used in these experiments. Only scarcely, results were applied to the ultrashort regime, and only theoretically in [11] or experimentally to the case of noble metals in [15,20].

This study is here applied to post-transition (Al) and transition (W, Ni, Cu) metals with diverse electronic structure, from Density of State (DOS) close to the free electron gas model to more complex DOS distribution with possibility of excitation of bound d-band transitions.
The paper is organized as follows. After description of the experimental configuration and diagnostics in section 2, we introduce briefly the theoretical background in section 3 . Experimental results made of reflectivity evolution as a function of fluence are further presented and discussed in section 4. Critical assessment of the experimental and simulation data allows us to retrieve the optical transient properties of the four different metals averaged over the pump pulse duration. These results shed light on crucial parameters driving laser energy coupling at the surface of metals and their optical response like the effective electron collision rate.

\section{Experiments, diagnostics, methodology and assump- tions}

Beamline 5a of ASUR platform [21] (800 nm, 30 fs FWHM, $\Delta \lambda \cong 760-840 \mathrm{~nm}$, linear polarization) is used to explore laser excitation of metals in the single-shot regime and at normal incidence below and above ablation $(0.1-15$ $\mathrm{F}_{\text {th }}$, with $\mathrm{F}_{\text {th }}$, the laser-induced ablation threshold fluence). This range of laser parameters gives a convenient tool for probing laser heating of metals in strong non-equilibrium conditions and under various situations of energy coupling and relaxation in the material. In particular, the energy of the laser pulse reflected by the sample during the interaction is carefully measured by means of photodiodes $\left(\mathrm{Ph}_{\mathrm{R}}\right.$ and $\mathrm{Ph}_{\text {inc }}$ for controlling the reflected and incident energy respectively, see the experimental setup in figure 1) to provide the evolution of the reflectivity integrated over the pulse duration as a function of applied fluence.

Because of the shortness of the pulse duration (30 fs) used in the experiments, there is no time for significative expansion of the free electron gas plasma developing at the surface of the samples and no hydrodynamical consideration has to be accounted for in our analysis. We experimentally verified that assumption by replacing the photodiode $\mathrm{Ph}_{\mathrm{R}}$ by a CCD beam analyzer camera to monitor the reflected beam. This verification was done on a fused silica wedged sample. We observed that whatever the incident 
energy (below and above the threshold $\mathrm{F}_{\text {th }}$ ) the reflected beam is captured at the same location (within beam pointing stability fluctuations) and its spatial shape and distribution is kept homothetically identical (see figure 1, left top) ensuring that significant plasma expansion does not occur at the pulse time scale.

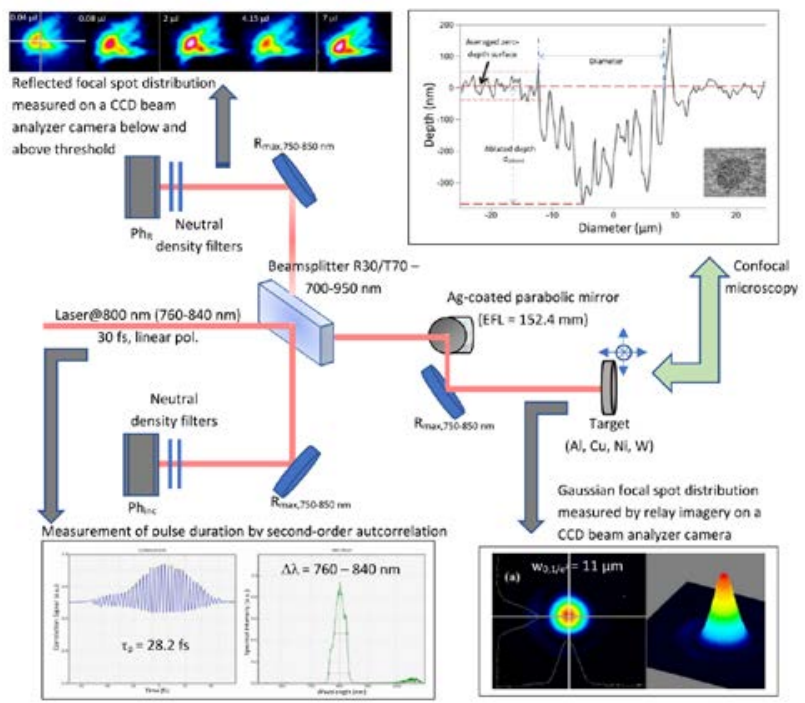

Fig. 1 Experimental setup (centre) and in-situ (grey arrows) and ex-situ (green arrow) diagnostics. In-situ diagnostics comprises reflectivity measurements using calibrated photodiodes and laser optical diagnostics. Ex-situ diagnostic consists of confocal microscopy to characterize laser-induced ablation craters on the four samples (done with Leica DCM 3D with axial (down to $<2$ $\mathrm{nm}$ ) and lateral (down to $0.14 \mu \mathrm{m}$ ) resolution). The target made of metallic thick samples ( $\mathrm{Al}, \mathrm{Cu}, \mathrm{Ni}$ and $\mathrm{W}$ ) is located in the focal plane of the parabolic mirror in which the beam waist $\mathrm{w}_{0}$ is measured (low corner, right).

The characteristics of the four different metallic samples are listed in table 1 . Using post-mortem diagnostic (confocal microscopy) and classical diameter-regression technique [22], the ablation threshold fluence was determined for the four different metals as a function of ultrashort pulse duration (see values in table 1) [3]. Moreover, we measured the reflected energy of the samples at extremely low fluence ( $<<\mathrm{F}_{\text {th }}$, unperturbed state) using the collimated beam. This allows us to retrieve the optical and electrical characteristics of the samples in the unperturbed state to fix the reference initial values before applying laser excitation (see table 1). To do that, note that the geometry of our measurement does monitor only a small collecting numerical aperture (no use of integrating spheres) and so it only accounts for the energy specularly reflected by the samples. The contribution of the scattered energy reflected by the samples (especially important for $\mathrm{Al}$ and $\mathrm{Cu}$ samples for which we measured a large surface roughness, see table 1) was calculated and added to the measurement in order to define the reflectivity of the samples in the unperturbed state $\left(R_{\text {material }}\right.$ in table 1$)$. This was done using the approach estimating the reflectivity scattered by a rough surface (with $\mathrm{Ra}$ characteristics) to calculate the reflectivity of a perfectly smooth surface $\mathrm{R}_{\text {material }}[23,3,4]$. Those values are in very good agreement (within $<2 \%$ ) with measured, tabulated or calculated reflectivity values available in literature. A similar strategy was also implemented at higher excitations considering that no changes in the surface morphology occurred at the time scale of interest (corresponding to the monitoring of the reflectivity measurement integrated over the laser pulse duration $\mathrm{R}_{\text {measured }}$ ) because of the short duration of the laser pulse used in this study.

Table 1. Characteristics and surface parameters of the samples, and their laser-induced ablation thresholds $\mathrm{F}_{\text {th }}$ experimentally determined [3]. *Literature reflectivity data (either calculated or measured in similar conditions): see [3] and references therein. The roughness Ra parameter has been measured using an AFM microscopy system (PSIA XE-100, Park Systems). The uncertainty on the measurement of reflectivity has been determined being $\mathrm{U}_{\text {mes }}= \pm 0.012[4]$.

\begin{tabular}{|c|c|c|c|c|}
\hline Material & $\mathrm{Al}$ & $\mathrm{Cu}$ & $\mathrm{Ni}$ & $\mathrm{W}$ \\
\hline $\begin{array}{c}\text { Quality/Purity } \\
\text { (from Goodfel- } \\
\text { low) }\end{array}$ & $\begin{array}{c}\text { AL000651, } \\
99.999 \% \\
\text { purity }\end{array}$ & $\begin{array}{c}\text { CU000750, } \\
99.9 \% \\
\text { purity }\end{array}$ & $\begin{array}{c}\text { Ni000624, } \\
99.99+\% \\
\text { purity }\end{array}$ & $\begin{array}{c}\text { W000375, } \\
99.95 \% \\
\text { purity }\end{array}$ \\
\hline $\begin{array}{c}\text { Thickness/Size } \\
(\mathrm{mm})\end{array}$ & $0.5 / 25 \times 25$ & $1 / 10 \times 10$ & $3.2 / 25 \times 25$ & $2 / 10 \times 10$ \\
\hline $\begin{array}{c}\text { Roughness Ra } \\
(\mathrm{nm})\end{array}$ & 20 & 17 & 5 & 8 \\
\hline $\begin{array}{c}\mathrm{R}_{\text {measured }} \\
(+/-0.012)\end{array}$ & 0.773 & 0.908 & 0.682 & 0.497 \\
\hline $\begin{array}{c}\mathrm{R}_{\text {material }} \\
(+/-0.012)\end{array}$ & 0.853 & 0.975 & 0.686 & 0.505 \\
\hline \begin{tabular}{c}
$\mathrm{R}_{\text {litterature,800nm }}{ }^{*}$ \\
\hline $\mathrm{F}_{\text {th }}\left(\mathrm{J} / \mathrm{cm}^{2}\right)$
\end{tabular} & 0.868 & 0.962 & 0.69 & 0.495 \\
\hline
\end{tabular}

\section{Modelling}

In view of benchmarking the laser-material interaction in a wide range of excitation fluences and to provide important information for its further simulation and prediction, we use simplified but justified assumptions. Firstly, in the following reflectivity calculations, we neglect any change of the lattice temperature over the pulse time scale with respect to its initial (room) temperature. This is justified by the characteristic time of electron-ion energy transfer time being in the picosecond domain for all considered materials [24, see also table 2] so on a time much superior to the pulse duration (30 fs). Moreover, we assume instantaneous thermalization of the electron population and further use the electron temperature (as defined by eq. 1) to represent its average characteristics and the Drude-Lorentz formalism to calculate the reflectivity averaged over the pulse duration. Confrontation of those data to those measured allows us to determine for each material the evolution of the effective electron collision rate $v_{\text {eff }}$ as a function of applied fluence.

The electronic temperature is calculated from the measured reflectivity $R_{\text {measured (with the assumption } A}=1$ $\mathrm{R}_{\text {measured}}$ ) and the associated incident fluence $F_{\text {inc }}[24]$ using equation 1:

$$
\mathrm{T}_{e}(x, \mathrm{t})=\frac{4\left(1-\mathrm{R}_{\text {measured }}\right) \mathrm{F}_{\text {inc }}}{3 \mathrm{l}_{\mathrm{s}} \mathrm{n}_{\mathrm{e}}} \exp \left(-\frac{2 x}{\mathrm{l}_{\mathrm{a}}}\right)
$$

Where $x$ is the spatial coordinate along the optical axis, $\mathrm{n}_{\mathrm{e}}$ is the free electron population density which evolution as 
a function of excitation is followed and estimated using Density Functional Theory (DFT) calculations [25] and $l_{\mathrm{s}}$ is the skin depth. For each value of $\mathrm{R}_{\text {measured, the skin depth is }}$ calculated using the equation $\mathrm{l}_{\mathrm{s}}=\mathrm{c} / \omega \kappa$, where $\mathrm{c}$ is the speed of light, $\omega$ is the laser frequency and $\kappa$ is the imaginary part of the complex refractive index (as further determined in table 2). The value of $\mathrm{T}_{\mathrm{e}}$ considered in the following calculation corresponds to an average value of $\mathrm{T}_{\mathrm{e}}(\mathrm{x})$ along the skin depth. Note also that for simplification we do not consider any change of $\kappa$ (and skin depth) upon excitation.

For the electronic temperatures beyond the Fermi temperature (cold solid model), the effective electronic collision rate includes the electron-phonon $\left(v_{\mathrm{e}-\mathrm{ph}}\right)$ and electronelectron $\left(v_{\mathrm{e}-\mathrm{e}}\right)$ interactions: $v_{\mathrm{eff}}=v_{\mathrm{e}-\mathrm{ph}}+v_{\mathrm{e}-\mathrm{e}}$. The electronphonon collision rate is given by eq. 2 [26]:

$$
v_{e-p h}=\frac{3}{2} C_{\omega} \frac{k_{B} T_{i}}{\hbar}
$$

Where $k_{B}$ stands for the Boltzmann constant, $\hbar$ is the reduced Planck constant and $C_{\omega}$ is a proportionality coefficient determined for each material using the reflectivity values $\mathrm{R}_{\text {material }}$ from table 1 . For the unexcited cold solid at room temperature $(300 \mathrm{~K})$, electron-phonon collisions dominate [12] allowing us to estimate the initial effective electron collision in concordance with the reflectivity of the material measured in this interaction regime. Doing so, the values of $v_{\mathrm{e}-\mathrm{ph}}$ related to the reflectivity $\mathrm{R}_{\text {material }}$ (unperturbed state) are listed in table 2. The complex index deduced from such measurements made with large spectral bandwidth (760-840 nm) femtosecond pulses is also retrieved and compared to literature data available at $800 \mathrm{~nm}$. Agreement is very good for nickel and tungsten, correct for aluminium and intermediate for copper because of the large surface roughness Ra measured for these two last materials which affects the precision of the value of reflectivity experimentally measured.

In our calculation, describing the evolution of the reflectivity during the 30 -fs pulse, the lattice temperature and the electron-phonon collision rate are considered unchanged and equal to the unperturbed values. The electronelectron collision frequency, which dramatically changes upon excitation, is given by eq. 3 [11]:

$$
\frac{1}{\tau_{\varepsilon \theta}\left(E, T_{e}\right)}=\frac{\pi^{2} \sqrt{3} \omega_{p}}{128 E_{F}^{2}} \times \frac{\left(\pi k_{B} T_{e}\right)^{2}+\left(E-E_{F}\right)^{2}}{1+\exp \left(-\frac{E-E_{F}}{k_{B} T_{e}}\right)}
$$

$E_{F}$ being the Fermi energy, $E$ the electron energy, $\omega_{\mathrm{p}}$ the plasma frequency, and $\mathrm{T}_{\mathrm{e}}$ is the electronic temperature estimated with eq. 1.

Knowing the evolution of the effective collision frequency as a function of the fluence, we use the DrudeLorentz model to calculate the associated reflectivity. The evolution of the permittivity as a function of the collision frequency is given by equation 4 [27]:

$$
\begin{gathered}
\varepsilon=\varepsilon_{r}+i \varepsilon_{i}=\varepsilon_{\text {intra }}+\varepsilon_{\text {inter }} \\
=\left[1-\frac{f_{0} \omega \frac{\pi}{p}}{\omega(\omega-i v)}\right]_{D}+\left[\sum_{j=1}^{k} \frac{f_{j} \Omega_{p}^{3}}{\left(\omega_{j}^{3}-\omega^{2}\right)+i \omega \Gamma_{j}}\right]_{L}
\end{gathered}
$$

The first term $\varepsilon_{\text {intra }}$ corresponds to the Drude model and is associated with free-electron intraband transitions with the oscillator strength $f_{0}$, the collision rate $v_{\text {eff }}$, and $\omega$ the laser frequency. The second term $\varepsilon_{\text {inter }}$ corresponds to the Lorentz component where $f_{j}, \Omega_{p}\left(=ل_{f_{0} \times \omega_{p}}\right), \omega_{j}$, and $\Gamma_{j}$ are, respectively, the oscillator strength, the plasma frequency, and the scattering rate of the harmonically bound electrons that are excited via interband transition $\mathrm{j}$. All the parameters entering the calculations for the four metals at room temperature have been determined in [27] and are listed in the following table 2.

With the values of $\varepsilon_{r}$ and $\varepsilon_{i}$ calculated with eq. 4 , the reflectivity is given by:

$$
R=\frac{(1-n)^{2}+k^{2}}{(1+n)^{2}+k^{2}}
$$

In which the real (n) and imaginary (k) parts of the complex refraction index are classically defined by:

$$
n=\sqrt{\frac{\varepsilon_{y}+\sqrt{\varepsilon_{T}^{2}+\varepsilon_{i}^{2}}}{2}} \text { and } k=\sqrt{\frac{-\varepsilon_{y}+\sqrt{\varepsilon_{F}^{2}+\varepsilon_{i}^{2}}}{2}}
$$

Table 2. Parameters (in eV when relevant) applied in the calculation of the reflectivity with the Drude-Lorentz model, from [27]. *For $\mathrm{Cu}$, the reflectivity data was only retrieved using 1.9 free electron per atom as issued in [25]. In green, material data calculated and issued from literature or inferred from our experiments. Following excitation and formation of dense plasma yielding material ablation, the energy transfer time between the elec-

\begin{tabular}{|c|c|c|c|c|}
\hline Parameters & Al & $\mathbf{C u}$ & $\mathbf{N i}$ & $\mathbf{W}$ \\
\hline$\hbar \omega_{p}$ & 14.98 & $14.91^{*}$ & 15.92 & 13.22 \\
\hline$f_{0}$ & 0.523 & 0.575 & 0.096 & 0.206 \\
\hline$f_{1}$ & 0.227 & 0.061 & 0.100 & 0.054 \\
\hline$\Gamma_{1}$ & 0.333 & 0.378 & 4.511 & 0.530 \\
\hline$\omega_{1}$ & 0.162 & 0.291 & 0.174 & 1.004 \\
\hline$f_{2}$ & 0.050 & 0.104 & 0.135 & 0.166 \\
\hline$\Gamma_{2}$ & 0.312 & 1.056 & 1.334 & 1.281 \\
\hline$\omega_{2}$ & 1.544 & 2.957 & 0.582 & 1.917 \\
\hline$f_{3}$ & 0.166 & 0.723 & 0.106 & 0.706 \\
\hline$\Gamma_{3}$ & 1.351 & 3.213 & 2.178 & 3.332 \\
\hline$\omega_{3}$ & 1.808 & 5.300 & 1.597 & 3.580 \\
\hline$f_{4}$ & 0.030 & 0.638 & 0.729 & 2.590 \\
\hline$\Gamma_{4}$ & 3.382 & 4.305 & 6.292 & 5.836 \\
\hline$\omega_{4}$ & 3.473 & 11.18 & 6.089 & 7.498 \\
\hline$v_{\text {e-ph }}\left(\mathrm{s}^{-1}\right)$ & $5.65 \times 10^{14}$ & $3 \times 10^{13}$ & $1.0 \times 10^{14}$ & $1.7 \times 10^{14}$ \\
\hline$E_{F}(\mathrm{eV})$ & 10.8 & 7.05 & 11.7 & 9.2 \\
\hline $\begin{array}{c}\mathrm{n}+\mathrm{i \kappa} \\
\text { (this work) }\end{array}$ & $\begin{array}{c}3.234+ \\
\text { i8.372 }\end{array}$ & $\begin{array}{c}0.305+ \\
\text { i6.862 }\end{array}$ & $\begin{array}{c}2.432 \\
+\mathrm{i} 4.379\end{array}$ & $\begin{array}{l}3.648+ \\
\text { i2.8075 }\end{array}$ \\
\hline $\begin{array}{c}n+\mathrm{i \kappa} \\
\text { (literature) }\end{array}$ & $\begin{array}{c}2.767+ \\
\text { i8.354 } \\
{[27]} \\
\end{array}$ & $\begin{array}{c}0.2535+ \\
\text { i5.013 } \\
{[28]} \\
\end{array}$ & $\begin{array}{c}2.43+ \\
\mathrm{i} 4.31 \\
{[29,30]} \\
\end{array}$ & $\begin{array}{c}3.67+ \\
\text { i2.68 } \\
{[29]} \\
\end{array}$ \\
\hline $\begin{array}{l}\text { Energy trans- } \\
\text { fer coupling } \\
\text { time (ps) }\end{array}$ & 2.2 & 5.1 & 4.4 & 16.7 \\
\hline
\end{tabular}
tron and lattice sub-systems is estimated from $\tau_{\text {coupling }} \approx \frac{M}{m_{\theta} v_{e i}}$ [24], where $v_{\mathrm{ei}}$ is the electron-ion collision frequency ( $v_{\text {ei }} \cong v_{\text {eff }} \cong \omega_{\mathrm{p}}$ is approximated for the above calculations in ablation conditions) and $\mathrm{M}$ stands for the atomic mass of the material and $m_{e}$ is the electron mass.

\section{Results and discussion}

For each material studied, the evolution of the reflectivity is calculated as a function of the incident fluence using eqs 1-6. On figure 2, the reflectivity calculated with the Drude (first term of eq. 4 only) and Drude-Lorentz model 
(both terms of eq. 4) is plotted with the measured reflectivity for each metal and for a pulse duration of $30 \mathrm{fs.}$

For the lowest fluences, therefore the lowest electron temperatures, the Drude-Lorentz model is in good agreement with the experimental results (with differences $<<$ $\left.\mathrm{U}_{\text {mes}}\right)$ for the four metals studied while the Drude model fails. At this low level of excitation, the band structure of the metals is still conserved, and interband transitions play an important role in the optical response of the materials. Then, when the incident fluence is increasing, even significantly above the ablation threshold fluence, the reflectivity $\mathrm{R}_{\text {measured }}$ starts to decrease for aluminum, copper and nickel but increases for tungsten.
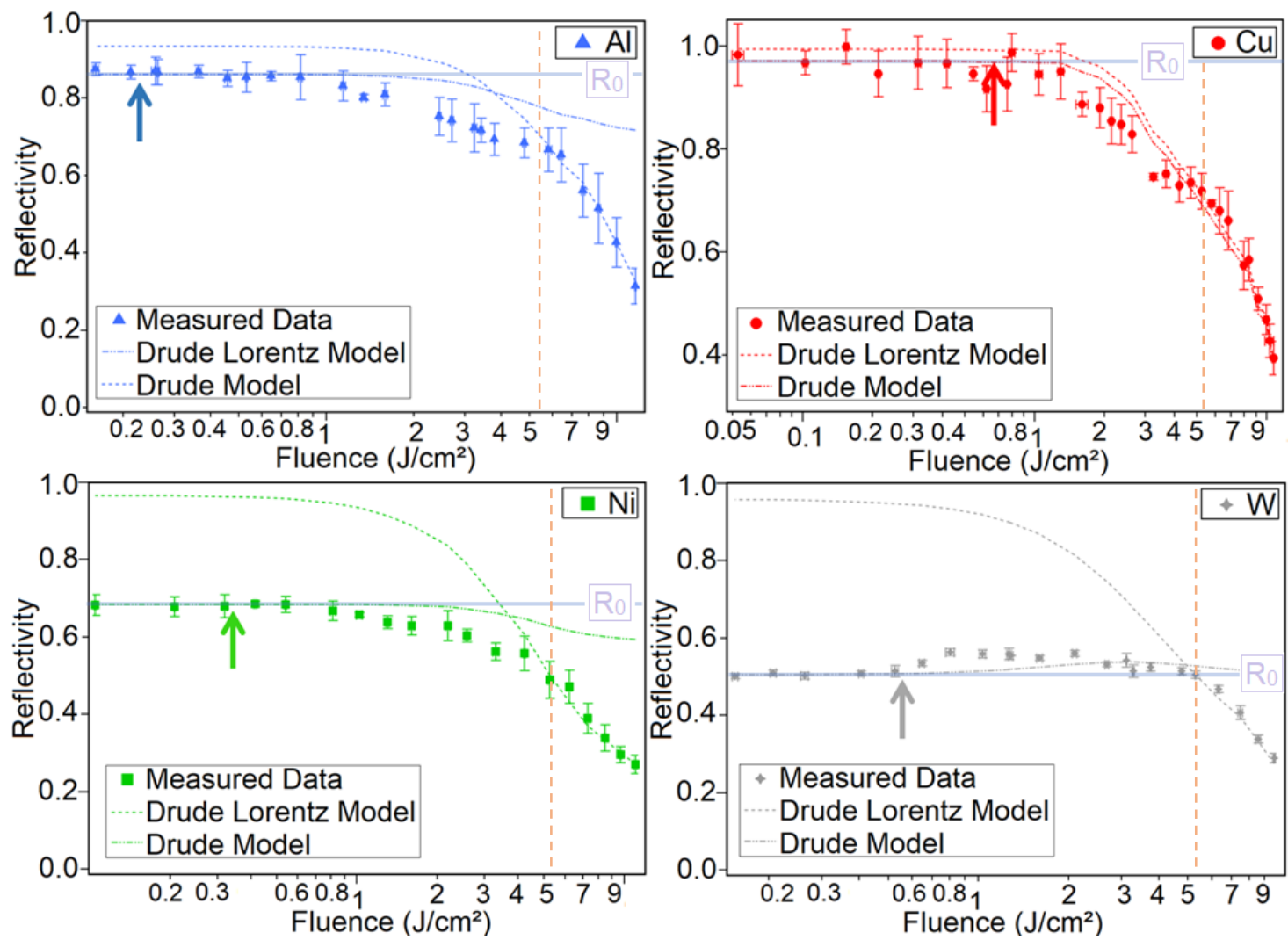

Fig. 2 Evolution of the reflectivity calculated with the Drude (first term of eq. 4) and Drude-Lorentz (both terms of eq. 4) model, compared to the experimental results as a function of the incident fluence. Pulse duration: $30 \mathrm{fs}$. The vertical error bars correspond to the standard deviation over 8 different measurements, and the horizontal error bars correspond to the shot-to-shot energy fluctuations measured on the incident photodiode. The colored arrows indicate the laser-induced ablation threshold fluence $F_{\text {th }}$ for each material. The orange dotted vertical line marks the fluence above which the beam focal plane starts to move towards the focusing parabolic mirror due to nonlinear propagation effects in air (see [3]). $\mathrm{R}_{0}$ corresponds to the reflectivity of the samples in the unperturbed state.

In the case of tungsten, this particular behaviour is ascribed to the presence of a low-density-of-state region around the Fermi energy separating lower and upper high density-of-state zones of the electron 3d-band [4,25,31]. Indeed, this low-density region acts as a bandgap hindering laser photon coupling to the material and resulting in an increase of the reflectivity. Only at high applied fluence (> $\sim 2.5 \mathrm{~J} / \mathrm{cm}^{2}$, see figure 2) the influence of this bandgap can be cancelled and photon coupling can efficiently be developed in high-density high-energy DOS region (on upper edge of the 3d-band and 4s-intraband population) yielding a large increase of the absorption.

For the highest fluences, a significant decrease in reflectivity is measured for the four metals. The absorption of the leading edge of the laser pulse leads to a strong rise of the electronic temperature and to the creation of a free electron plasma. In these conditions of high excitation yielding rapidly the formation of a dense plasma at the surface of the materials, the influence of band structure and of its bound electron component becomes negligible. The Drude model, which incorporates the contribution of the free electron population only, thus allows us to describe the interaction in very good agreement (with differences $<<U_{\text {mes}}$ ) with the experimental measurements for the four metals (see figure $2)$. We note that discrepancies between experiments and modelling are mostly observed at intermediate fluences ( $1-4 \mathrm{~J} / \mathrm{cm}^{2}$ ). In this irradiation regime, the electron characteristics evolves significantly and rapidly during the pulse in the laser-heated material, with transition between a cold solid material (well described by the Drude-Lorentz model) to a free-electron plasma rapidly developing at the material surface (well described by the Drude model). As an example of improvement of the theoretical description, it would be beneficial to include in the description of the DrudeLorentz model (eq. 4) the progressive change incurred by the bound term due to the development of the laser excitation to better handle the progressive destruction of the band structure.

Finally, we also observe that a satisfactory agreement is obtained for both models in the case of $\mathrm{Cu}$ on the whole range of laser excitation fluence, in comparison to the other three metals studied. We attribute this observation to the 
importance of the delocalized electrons in the optical response of $\mathrm{Cu}$ whatever the level of excitation and in particular at low fluence for which the reflectivity is close to the reflectivity of a free-electron dense plasma conveniently described by the Drude model.

Using the approach described in section 3, the evolution of the effective collision frequency $v_{\text {eff }}$ averaged over the pulse duration (and spatially over the skin depth $l_{\mathrm{s}}$ ) is also plotted on figure 3 as a function of the incident fluence. This parameter is of major importance for the description of the evolution of the optical parameters and energy redistribution and transport of a metal following femtosecond laser excitation. In first approximation, note that we do not consider any influence of the nonlinear propagation effects developing in air in front of the target [3] for the building of the data set at high excitation fluence $\left(F>\sim 6 \mathrm{~J} / \mathrm{cm}^{2}\right)$ shown in figure 3.

From figure 3, it can be seen a swift increase of the effective electron collision frequency at high excitations $(F \geq$ $\left.F_{\text {th }}\right)$ in relation with the formation of dense plasma at the surface of the materials. This is due to the prompt growth of the electron-electron collision frequency consecutive of the increase of the electron temperature and of the importance of delocalized free electron transitions. This observed evolution retrieved from thorough comparison between experiments qualitatively confirms and clarifies (here for four metals) what was theoretically predicted in seminal previous works and calculations (see for instance $[12,31])$.

For aluminum and copper (and qualitatively for nickel), which are considered in [12,13] for longer pulse durations ( $>100 \mathrm{fs}$ ), we also observe a very good quantitative agreement concerning the range of value reached. Moreover, we also set into evidence that the increase of the effective electron collision frequency observed at rather high excitations ( $\mathrm{F} \geq \mathrm{F}_{\mathrm{th}}$ ) is in correlation with the prompt augmentation of the electron-electron interactions [13] while electron-phonon component $v_{\mathrm{e}-\mathrm{ph}}$ does not change significantly. This last result is linked to insufficient time (30-fs pulse) to have significant lattice heating of the material during the pulse. We also note that this increase above the initial (at rest) $v_{0, \text { eff }}$ value does occur at rather high excitation for aluminum $\left(\cong 4-5 \times F_{\text {th }}\right)$ while for all materials the increase initiates close to the ablation threshold. Moreover, the absence of strong changes of the effective collision frequency $v_{\text {eff }}$ observed at low excitations for all materials is in line with the limited perturbations incurred by the electronic band structure until reaching the ablation threshold fluence. Nevertheless, for some materials, as shown for tungsten, those perturbations have also been proven influential on the optical transient properties with the increase of reflectivity (with $\Delta R / R_{\text {unperturbed state }} \cong 5 \%$, see figure 2 ) for fluences slightly above the ablation threshold. Finally, as shown elsewhere [11,14-16], reduction of the thermalization time of the electron gas with further consequence on energy exchange with the lattice is expected at high excitation ( $\mathrm{F} \geq \mathrm{F}_{\text {th }}$ ) for the four studied materials because of the predominance of electron-electron interactions with respect to what is observed at lower excitations.

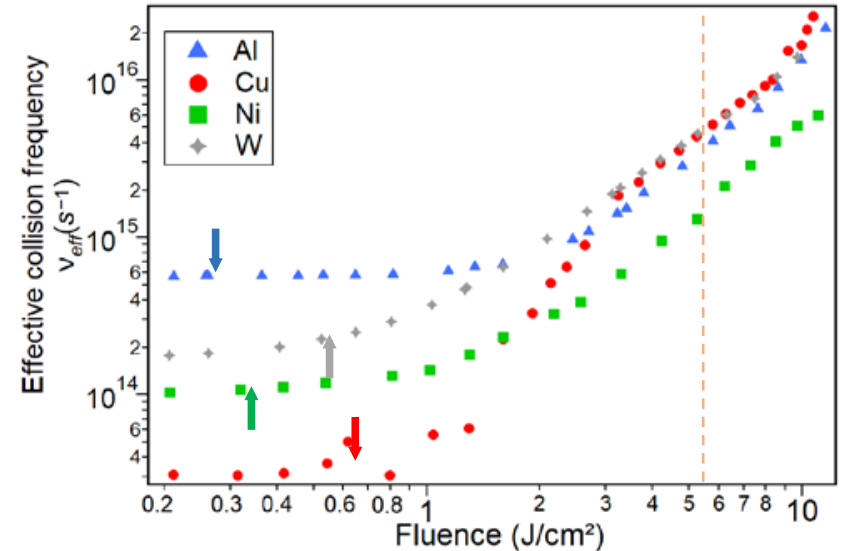

Fig. 3. Evolution of the effective collision frequency $v_{\text {eff }}$ as a function of the incident fluence and for the four metals studied. Pulse duration: $30 \mathrm{fs}$. The arrows and orange dot line have same meaning as in figure 2. To get those data, using the reflectivity determined experimentally, and the equation 1 , the electronic temperature $T_{e}$ is determined. Then, the value of $T_{e}$ is injected into eq 3 to obtain the effective collision frequency with the formula $v_{\text {eff }}=v_{\text {e-ph }}+v_{\text {e-e. }}$. Note that the initial value of $v_{\text {e-ph }}$ was calculated using the Drude-Lorentz model to take into account the importance of the band structure at low excitation.

Determination of the effective electron collision rate on such wide range of excitations below and much above the ablation threshold is highly rewarding. As examples, it can serve to improve the description of fundamental aspects of the interaction, e.g. for better handling equilibration time of electron population sub-system or transient optical properties and high frequency conductivity of the excited medium, and so physical energy exchanges, relaxation and transport phenomena yielding to damage and ablation. This should be in particular performed in the context of more detailed and elaborated modelling approaches considering the evolution of relevant material parameters as a function of laser excitation available from literature.

On an applied perspective, this knowledge should have many interests in surface structuration or micro-machining applications.

\section{Conclusion}

After describing our methodology based on a thorough comparison between experimental and calculated data representative of laser-matter interaction, we apply this strategy to four metals exposed to ultrashort 30-fs single laser pulses and a wide range of excitations $\left(\cong 0.1-10 \mathrm{~J} / \mathrm{cm}^{2}\right)$ illustrative of many situations of laser-matter interaction. In particular, we succeed in determining a crucial parameter characterizing and driving the interaction, i.e. the effective electron collision rate $v_{\text {eff }}$ as a function of applied fluence. The experimental determination of this parameter will help to work out better predicting laser - matter interaction models, to better handle or anticipate dedicated strategy to enhance and control energy deposition and to boost the development of optimized laser-based processes, here more specifically focused on ultrashort laser micro-machining of metals.

On a more fundamental aspect, it was shown the high relevance of the Drude-Lorentz model to account for the evolution of the reflectivity and effective collision frequency of the materials in the low-fluence range $\left(\sim \leq 1 \mathrm{~J} / \mathrm{cm}^{2}\right)$ 
while Drude model appears to be well adapted in the highfluence range $\left(\sim \geq 4 \mathrm{~J} / \mathrm{cm}^{2}\right)$. In the intermediate fluence range ( $1-4 \mathrm{~J} / \mathrm{cm}^{2}$ ), one should improve the DrudeLorentz description in which the progressive vanishing of the bound term due to the progress of excitation should be accounted for in a different quantitative way depending on the material (as suggested for instance by the compared cases of tungsten and nickel).

\section{Funding}

Financial support of the ASUR platform was provided by the European Community and LaserLab Europe program (Project Laserlab-Europe Nos. EU-H2020 654148 and 871124), Ministry of Research and High Education, Region Provence-Alpes-Côte d'Azur, Department of Bouches-du-Rhône, City of Marseille, CNRS, and AixMarseille University.

\section{Acknowledgements}

T. Genieys acknowledges the support of DGA - Direction Générale de l'Armement (Ministry of Defense), AixMarseille University and Ministry of Research and higher Education for his Ph'D grant.

\section{References}

[1] J. Cheng, C.-S. Liu, S. Shang, D. Liu, W. Perrie, G. Dearden, and K. Watkins: Opt. Laser Technol., 46, (2013) 88.

[2] B.N. Chichkov, C. Momma, S. Nolte, F. von Alvensleben, and A. Tünnermann: Appl. Phys. A, 63, (1996) 109.

[3] T. Genieys, M. Sentis, and O. Utéza: Adv. Opt. Technol., 9, (2020) 131.

[4] T. Genieys, M. Sentis, and O. Utéza: Appl. Phys. A 126, 263 (2020)

[5] P.T. Mannion, J. Magee, E. Coyne, G.M. O’Connor, and T.J. Glynn: Appl. Surf. Sci., 233, (2004) 275.

[6] D.A. Zayarny, A.A. Ionin, S.I. Kudryashov, S.V. Makarov, A.A. Kuchmizhak, O.B Vitrik, and Y.N. Kulchin: Laser Phys. Lett., 13, (2016) 076101.

[7] S.E. Kirkwood, A.C. Van Popta, Y.Y. Tsui, and R. Fedosejevs: Appl. Phys. A, 81, (2005) 729.

[8] J. Byskov-Nielsen, J.-M. Savolainen, M. Snogdahl Christensen, and P. Balling: Appl. Phys. A, 101, (2010) 97.

[9] A.Y. Vorobyev and C. Guo: Laser Photonics Rev., 7, (2013) 385.

[10] J. Bonse, S. Höhm, S. V. Kirner, A. Rosenfeld, and J. Krüger: IEEE J. Sel. Top. Quantum Electron., 23, (2017) 9000615.

[11] B.Y. Mueller and B. Rethfeld: Phys. Rev. B, 87, (2013) 035139.
[12] K. Eidmann, J. Meyer-ter-Vehn, T. Schlegel, and S. Hüller: Phys. Rev. E, 62, (2000) 1202.

[13] A. Suslova and A. Hassanein: Laser Part. Beams, 35, (2017) 415.

[14] B. Rethfeld, A. Kaiser, M. Vicanek, and G. Simon: Phys. Rev. B, 65, (2002) 214303.

[15] N. Del Fatti, C. Voisin, M. Achermann, S. Tzortzakis, D. Christofilos, and F. Vallée: Phys. Rev. B, 61, (2000) 16956.

[16] R.H.M. Groeneveld, R. Sprik, and A. Lagendijk: Phys. Rev. B 45, (1992) 5079.

[17] N.A. Inogamov, V.V. Zhakhovskii, S.I. Ashitkov, V.A. Khokhlov, Yu.V. Petrov, P.S. Komarov, M.B. Agranat, S.I. Anisimov, and K. Nishihara: Appl. Surf. Sci., 255, (2009) 9712.

[18] A. Blumenstein, E.S. Zijlstra, D.S. Ivanov, S.T. Weber, T. Zier, F. Kleinwort, B. Rethfeld, J. Ihlemann, P. Simon, and M. E. Garcia: Phys. Rev. B, 101, (2020) 165140

[19] J. Winter, S. Rapp, M. Schmidt, and H.P. Huber: Appl. Surf. Sci., 417, (2017) 2.

[20] C. Fourment, F. Deneuville, D. Descamps, F. Dorchies, S. Petit, and O. Peyrusse : Phys. Rev. B, 89, (2014) 161110(R).

[21] O. Utéza, P. Blandin, L. Charmasson, G. Coustillier, D. Grojo, A. Kabashin, M. Lebugle, N. Sanner, V. Tcheremiskine, M. Sentis, F. Légaré, and J.-C. Kieffer : EDP Sciences, UVX 2012, (2013) 01004.

[22] J.M. Liu: Opt. Lett. 7, (1982) 196.

[23] A.M. Prokhorov, V.I. Konov, I. Ursu, and I.N. Mihailescu: "Laser heating of metals", ed. by The Adam Hilger Series on Optics and Optoelectronics, (1990).

[24] E.G Gamaly, A.V. Rode, B. Luther-Davies, and V.T. Tikhonchuk: Phys. Plasmas, 9, (2002) 949.

[25] E. Bévillon, J.-Ph. Colombier, V. Recoules, and R. Stoian: Phys. Rev. B, 89, (2014) 115117.

[26] E.G. Gamaly and A.V. Rode: Prog. Quantum. Electron., 37, (2013) 215.

[27] A.D. Rakic, A.B. Djurisic, J.M. Elazar, and M.L. Majewski: Appl. Opt., 37, (1998) 5721.

[28] P. B. Johnson and R. W. Christy: Phys. Rev. B, 6, (1972) 4370.

[29] M.J. Weber: "Handbook of Optical Materials”, ed. by CRC Press (LLC, NYC, USA, 2003).

[30] D.R. Lide: CRC Handbook of Chemistry and Physics 2006-2007 87 ${ }^{\text {th }}$ edn. (CRC Press, London).

[31] E. Bévillon, R. Stoian, and J.Ph. Colombier: J. Phys.: Condens. Matter, 30, (2018) 385401.

(Received: June 10, 2021, Accepted: October 20, 2021) 